\title{
Mössbauer Study of Electronic Spin-Flip Processes in $\mathrm{NH} 4 \mathrm{Fe}(\mathrm{SO} 4) 2 \cdot 12 \mathrm{H} 2 \mathrm{O}$
}

\author{
Mørup, Steen; Thrane., N.
}

Published in:

Physical Review B Condensed Matter

Link to article, DOI:

10.1103/PhysRevB.4.2087

Publication date:

1971

Document Version

Publisher's PDF, also known as Version of record

Link back to DTU Orbit

Citation (APA):

Mørup, S., \& Thrane., N. (1971). Mössbauer Study of Electronic Spin-Flip Processes in NH4 Fe(SO4)2 $12 \mathrm{H} 2 \mathrm{O}$. Physical Review B Condensed Matter, 4(7), 2087-2091. https://doi.org/10.1103/PhysRevB.4.2087

\section{General rights}

Copyright and moral rights for the publications made accessible in the public portal are retained by the authors and/or other copyright owners and it is a condition of accessing publications that users recognise and abide by the legal requirements associated with these rights.

- Users may download and print one copy of any publication from the public portal for the purpose of private study or research.

- You may not further distribute the material or use it for any profit-making activity or commercial gain

- You may freely distribute the URL identifying the publication in the public portal

If you believe that this document breaches copyright please contact us providing details, and we will remove access to the work immediately and investigate your claim. 


\title{
Mössbauer Study of Electronic Spin-Flip Processes in $\mathrm{NH}_{4} \mathrm{Fe}\left(\mathrm{SO}_{4}\right)_{2} \cdot 12 \mathrm{H}_{2} \mathrm{O}$
}

\author{
S. Morup and N. Thrane \\ Laboratory of Applied Physics II, Technical University of Denmark, DK 2800 Lyngby, Denmark \\ (Received 9 July 1970; revised manuscript received 9 April 1971)
}

\begin{abstract}
Relaxation-broadened Mössbauer spectra of ferric alum have been obtained in the temperature range 85-250 $\mathrm{K}$, with 0-5-kG applied magnetic field. In fields above a few $\mathrm{kG}$ the spectra are only influenced by hyperfine fields fluctuating parallel to the applied-field direction. In low applied fields the spectra are also influenced by components of the hyperfine field fluctuating perpendicular to the applied-field direction, thereby inducing transitions between the nuclear sublevels. In the case of high applied fields, a simple model permits the determination of the spin-correlation time. In 5-kG applied fields, the spin-correlation time was found to decrease from $1.6 \pm 0.15 \mathrm{nsec}$ at $85 \mathrm{~K}$ to $0.50 \pm 0.05 \mathrm{nsec}$ at $245 \mathrm{~K}$ due to spin-lattice interactions. It is shown that these results cannot be compared with the values obtained by highfrequency susceptibility measurements.
\end{abstract}

\section{INTRODUCTION}

Mössbauer spectra of paramagnetic compounds are often influenced by fluctuating hyperfine fields. This has been described in a number of publications. ${ }^{1-12}$ In undiluted ferric compounds a broadening of the lines can often be found. ${ }^{13-15}$ Sufficient dilution with aluminum or other nonmagnetic ions can result in the appearence of magnetically split spectra. ${ }^{16,17}$

The Mössbauer spectrum of $\mathrm{NH}_{4} \mathrm{Fe}\left(\mathrm{SO}_{4}\right)_{2} \cdot 12 \mathrm{H}_{2} \mathrm{O}$ (ferric alum) has been investigated by several authors. ${ }^{14-22}$ Highly magnetized samples exhibit magnetic splitting with anomalous line broadenings and line displacements. ${ }^{18-20}$ At room temperature the spectrum consists of a single broad line which in low fields becomes considerably narrower. ${ }^{14,15}$ In zero field the linewidth depends on the temperature in a complicated way. ${ }^{22}$

\section{EXPERIMENT}

Mössbauer spectra of polycrystalline $\mathrm{NH}_{4} \mathrm{Fe}$ $\left(\mathrm{SO}_{4}\right)_{2} \cdot 12 \mathrm{H}_{2} \mathrm{O}$ were obtained in order to examine the spin-flip processes. The temperature was varied from 85 to $250 \mathrm{~K}$. Magnetic fields in the range 0-5 kG were applied parallel and perpendicular to the $\gamma$-ray direction. A $10-\mathrm{mCi} \mathrm{Co}^{57}$ source in Pd was used. The absorbers were prepared with a thickness of $150 \mathrm{mg} / \mathrm{cm}^{2}$ and cooled immediately in order to prevent water absorption. During each measurement the absorber temperature was controlled to within $\pm 1 \mathrm{~K}$. The measurements were carried out with an ordinary constant-acceleration Mössbauer spectrometer with the multichannel analyzer in the PHA mode.

\section{THEORY}

\section{A. Mössbauer Line Broadening}

An increasing number of papers ${ }^{1-12}$ deal with the influence of fluctuating hyperfine fields on the Mössbauer spectra of paramagnetic compounds. The hyperfine interaction is generally described 4 by the Hamiltonian $\hat{H}=\overrightarrow{\mathrm{I}} \cdot \overrightarrow{\mathrm{A}} \cdot \overrightarrow{\mathrm{S}}$, where $\overrightarrow{\mathrm{S}}$ is the atomic spin, $\vec{I}$ is the nuclear spin, and $\vec{A}$ is a tensor, which depends on the crystal field as well as the nuclear properties. However, as demonstrated by Afanas'ev and other workers, ${ }^{23,24} \mathrm{a}$ rather small magnetic field $(\sim 100 \mathrm{G}$ in the case of $\mathrm{Fe}^{+3}$ ) is sufficient to secure the validity of an effective-field Hamiltonian, $\hat{H}=a \hat{S}_{k} \hat{I}_{k}=H_{\mathrm{hf}} \hat{I}_{k}$, where $a$ is a constant. In ferric alum this Hamiltonian must be valid as the field $H_{i}$ at one ferric ion arising from the neighboring iron ions is about $450 \mathrm{G}$ on the average. ${ }^{25}$

In ferric alum diluted with aluminum the crystal-field splitting is small, about $0.12 \mathrm{~K} .{ }^{26-28}$ It is reasonable to assume a splitting of the same order in the pure compound. Therefore, the crystal-field interaction is comparable to the magnetic dipole-dipole interaction between neighboring ferric ions. This means that the ionic spin, and hence the hyperfine field, will vary in direction according to the magnitude and direction of $H_{i}$ relative to the crystal-field axis. If a magnetic field $H_{0}$, large compared to $H_{i}$, is applied along the $z$ direction, the ionic spins will precess around this direction and the hyperfine field will only fluctuate along the same direction.

The ideal case where the hyperfine field fluctuates only parallel to the $z$ axis has been investigated theoretically by several authors. Wegener ${ }^{5}$ considers only short relaxation times $\left(10^{-9}-10^{-12}\right.$ sec) whereas other author ${ }^{1-4,7-12}$ also consider longer relaxation times. In the limit of fast relaxation all these theories give essentially the same result, namely, a broadening of the absorption lines proportional to $\tau\left\langle h^{2}\right\rangle_{\mathrm{av}} \nu^{2}\left(m_{e}, m_{g}\right)$. Here, $\tau$ is the spin-flip time or the spin-correla- 


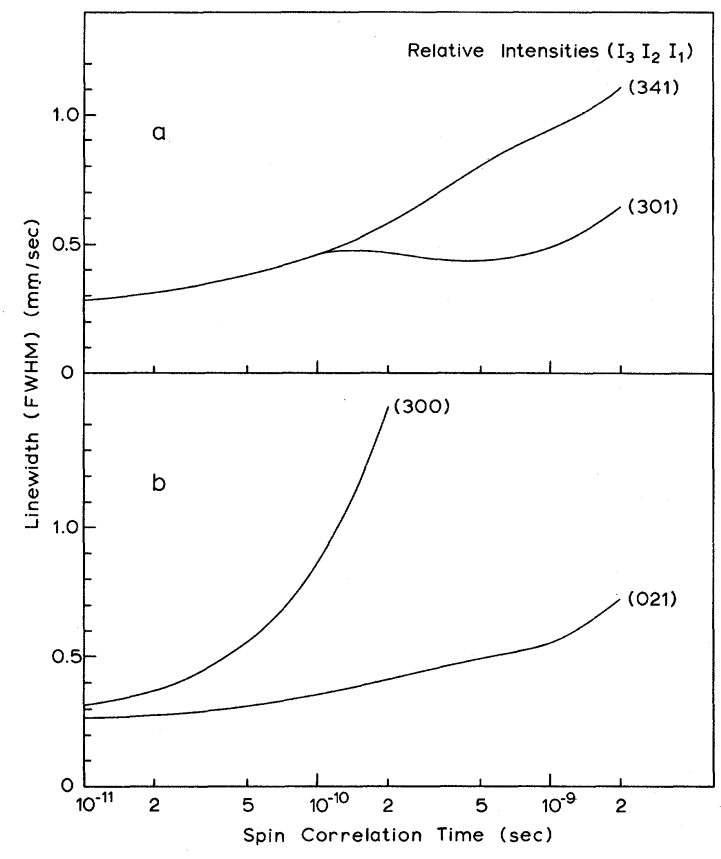

FIG. 1. Computed linewidth (FWHIM) as a function of the spin-correlation time for $\left\langle h^{2}\right\rangle_{2 \mathrm{v}}=162400 \mathrm{kG}^{2}$ and $\Gamma_{0}=0.25 \mathrm{~mm} / \mathrm{sec}$. Relative intensities of the three components are given in parentheses. (341) and (301) correspond to external magnetic field perpendicular and parallel to the $\gamma$-ray direction, respectively. (300) and (021) correspond to the two lines in the case of quadrupole splitting.

tion time, $h$ is related to the magnitude of the fluctuating hyperfine field, and $\nu\left(m_{e}, m_{g}\right)$ is the difference between the $z$ components of the nuclear magnetic moments of the initial state and the final state of the individual $\gamma$ transitions. The various theories differ at most in the definitions of $\left\langle h^{2}\right\rangle_{\mathrm{av}}$ and $\tau$.

In the present work we have applied the shortcorrelation-time approximation of van der Woudes theory ${ }^{8}$ as derived by van Zorge et al. ${ }^{9}$

Neglecting the average magnetic field $\left\langle\overrightarrow{\mathrm{H}}_{0}+\overrightarrow{\mathrm{H}}_{\mathrm{hf}}\right\rangle_{\text {av }}$ and the electric field gradient at the nucleus, the absorption spectrum will consist of three equally positioned Lorenzian lines corresponding to the different nuclear transitions. The linewidths are

$$
\Gamma=\Gamma_{0}+\left\langle h^{2}\right\rangle_{\mathrm{av}} \nu^{2}\left(m_{e}, m_{g}\right) \tau,
$$

where $\left\langle h^{2}\right\rangle_{\mathrm{av}}$ is the average value of the squared hyperfine field. As $H_{\mathrm{hf}}=a S_{z}$ and $S=\frac{5}{2}$, we get

$$
\left\langle h^{2}\right\rangle_{\mathrm{av}}=a^{2}\left\langle S_{k}^{2}\right\rangle=a^{2} \frac{35}{12} \quad .
$$

$\tau$ is the spin-correlation time and $\Gamma_{0}$ is the experimental linewidth in the limit of very fast fluctuating fields. The relative integral inten- sities of the three Lorentzians are 3:4:1 and 3:0:1 for the applied magnetic field perpendicular and parallel to the $\gamma$-ray direction, respectively.

The total linewidth, full width at half-maximum (FWHM), of an absorption line has often been connected to the correlation time $\tau$. In Fig. 1(a) is shown the calculated total linewidth (FWHM) for parallel and perpendicular field as a function of $\tau$ for $\Gamma_{0}=0.25 \mathrm{~mm} / \mathrm{sec}$ and $\left\langle h^{2}\right\rangle_{\mathrm{av}}=162400 \mathrm{kG}^{2}$ $\left(H_{\mathrm{hf}}=590 \mathrm{kG}\right.$ for $\left.S_{z}=\frac{5}{2}\right)$. In parallel field the linewidth is a decreasing function of $\tau$ for 0.15 $\leqslant \tau \leqslant 0.45 \mathrm{nsec}$. In the perpendicular case the linewidth is an increasing function of $\tau$ as expected, but the slope is not pronounced. Hence a fitting procedure is necessary when one wants to derive the spin-correlation time from a Mössbauer spectrum.

When a quadrupole splitting is present and the magnetic field is fluctuating along the axis of an axially symmetric electric field gradient, the broader line depends in a more simple way on the relaxation time [Fig. 1(b)].

In a previous paper ${ }^{29}$ we have discussed the shape of the Mössbauer spectrum as a function of the applied field $H_{0}$. For $H_{0} \gtrsim 1 \mathrm{kG}$, the absorption line consists of broad and narrow components in agreement with the theories mentioned above. The narrowest component, arising from the $\pm \frac{1}{2} \rightarrow \mp \frac{1}{2}$ nuclear transitions is responsible for the relatively small linewidth. When the applied field is decreased, the narrow component gradually disappears. It was proposed that magnetic fields fluctuating perpendicularly to the direction of the external field induce transitions between the nuclear sublevels. If the lifetime of a nuclear sublevel is not considerably longer than the nuclear Larmor period, the components of the Mössbauer spectrum will mix. ${ }^{7}$ Hence, the narrow component disappears resulting in an increased linewidth. Here we shall discuss this phenomenon a little further.

In a magnetic field $H_{1}$ fluctuating perpendicularly to the direction of a large constant field, the transition rate $P$ for transitions between nuclear sublevels is proportional to $H_{1}^{2}$. Neglecting the crystal-field splitting, $H_{1}$ is, as a good approximation, equal to the component of the hyperfine field $H_{\mathrm{hf}}$ perpendicular to the direction of the applied field $H_{0}$. The ionic dipole-dipole field $H_{i}$ is responsible for the time dependence of the orientation of $H_{\mathrm{hf}}$. For $H_{i}^{2} \ll H_{0}^{2}$, we find

$$
P \propto H_{\mathrm{hf}}^{2}\left\langle H_{i}^{2}\right\rangle_{\mathrm{av}} / H_{0}^{2}
$$

$P$ also depends on the detailed time variation of $H_{1}$. However, when only the amplitude of $H_{1}$ is changed, we see that if $P$ is comparable to the nuclear Larmor frequency at moderate applied fields $H_{0}$, an increase of $H_{0}$ by a factor of 3 will 
increase the lifetime of the nuclear sublevels so much that the transitions can hardly be observed. We believe that this is the explanation of the observed line narrowing.

The above explanation is supported by the fact that the line narrowing is obtained with smaller applied field in compounds diluted with aluminum, where $H_{i}$ is smaller. ${ }^{30}$ This suggests that the Mössbauer effect can give information about phenomena related to transverse relaxation. The detailed calculations still remain to be carried out.

\section{B. Spin-Flip Processes}

Transitions between the eigenstates of the atomic spin $\hat{S}_{z}$ are induced by spin-spin interactions and by spin-lattice interactions. Generally, the spin-correlation time measured by Mössbauer spectroscopy cannot be compared with the relaxation time measured in high-frequency susceptibility experiments, as the latter does not take into account spin-flip processes which leave the total magnetization of the sample unchanged. An example is the simultaneous transitions of two spins $S_{1}$ and $S_{2}$ induced by the operator $\hat{S}_{1}^{+} \hat{S}_{2}^{-}+\hat{S}_{1}^{-} \hat{S}_{2}^{+}$. These processes are energy conserving, contrary to other spin-flip processes caused by spin-spin interaction. Hence these processes are most important when the separation between the ionic

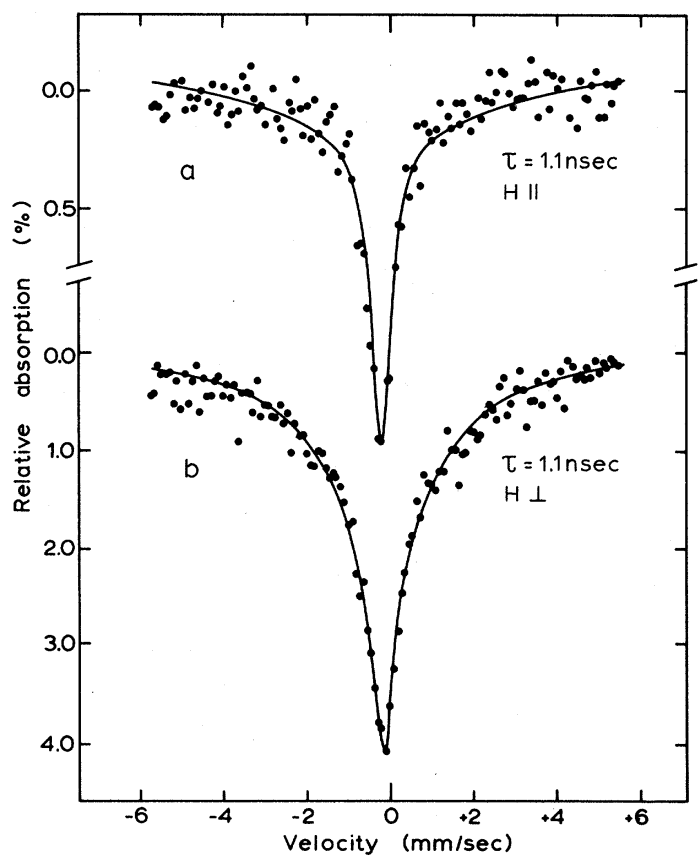

FIG. 2. Mössbauer absorption spectra of ferric alum at $120 \mathrm{~K}$ in a $5-\mathrm{kG}$ external field. (a) and (b) correspond to the external field parallel and perpendicular to the $\gamma$-ray direction, respectively. The solid lines represent computed spectra for $\tau=1.1 \mathrm{nsec}$.

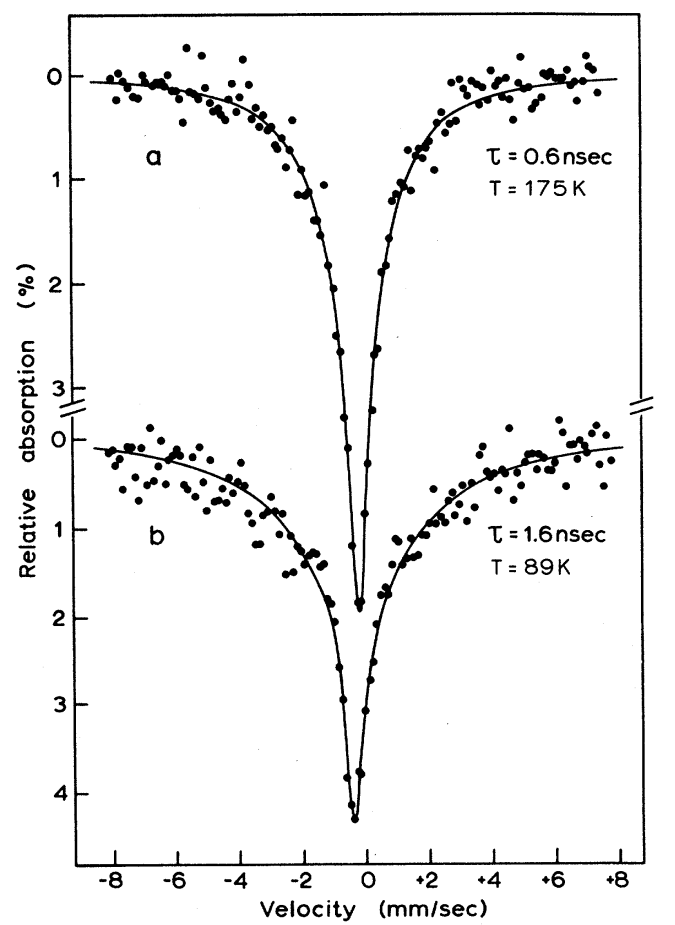

FIG. 3. Mössbauer absorption spectra of ferric alum in an external magnetic field of $5 \mathrm{kG}$ perpendicular to the $\gamma$-ray direction. (a) $T=175 \mathrm{~K}$; (b) $T=89 \mathrm{~K}$. The solid lines represent computed spectra for the indicated spin-correlation times.

levels is large relative to the dipole-dipole interaction energy. Having this in mind, one should describe the time-constant characteristic for the fluctuating hyperfine field not as a relaxation time but rather as a spin-correlation time or a spinflip time.

The spin-spin interaction is essentially temperature independent unless the separation between the ionic levels is comparable to the thermal energy giving rise to an unequal population of the levels, as in the case of $\mathrm{FeCl}_{3} \cdot 6 \mathrm{H}_{2} \mathrm{O}^{31}$ and ferric hemin. ${ }^{32}$ In contrast, spin-lattice interaction is normally strongly temperature dependent.

In ferric compounds the spin-lattice interaction is usually considered to be negligible compared to spin-spin interaction. However, in diluted compounds spin-spin processes are slowed down, and spin-lattice processes have been observed in a few cases. ${ }^{16,21,33}$ In a recent work by Svetozarov ${ }^{34}$ it was shown that the spin-flip rate due to interaction with the lattice vibrations is proportional to $T^{5}$ for temperatures well below the Debye temperature. In the high-temperature limit a proportionality to $T^{2}$ was found. Suzdalev et al. ${ }^{33}$ have calculated the detailed temperature dependence on basis of the Einstein model and the Debye model for the phonon spectrum. 


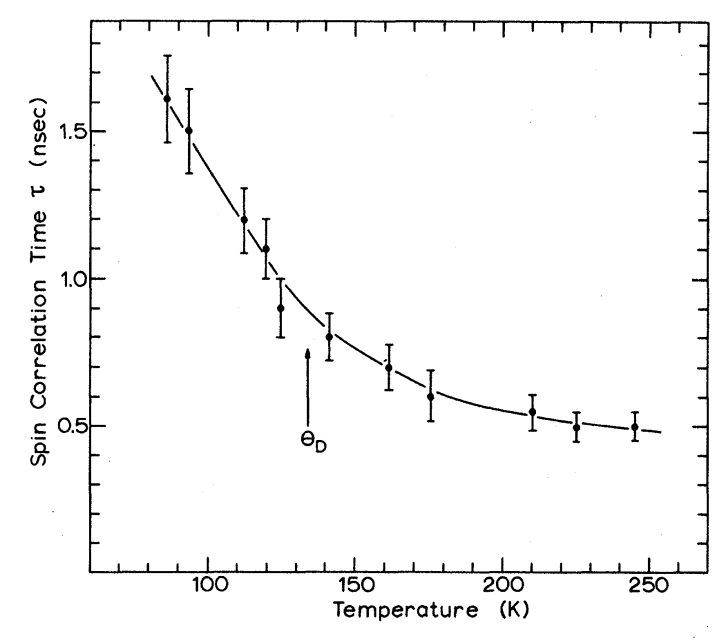

FIG. 4. Estimated values of the spin-correlation time $\tau$ of ferric alum as a function of temperature in a $5-\mathrm{kG}$ external field applied perpendicular to the $\gamma$-ray direction.

In the following we shall denote the time constants for spin-spin and spin-lattice interactions by $\tau_{s s}$ and $\tau_{s 1}$, respectively. The resulting spincorrelation time is denoted $\tau$.

\section{RESULTS}

A number of spectra have been obtained with the absorber in a magnetic field of $5 \mathrm{kG}$ and at temperatures in the range $85-250 \mathrm{~K}$. The spectra were computer fitted with the simple model described in Sec. III. In all fittings $\Gamma_{0}$ was 0.35 $\mathrm{mm} / \mathrm{sec}$ and $\left\langle h^{2}\right\rangle_{\mathrm{av}}=162400 \mathrm{kG}^{2}$. The value of $\left\langle h^{2}\right\rangle_{\text {av }}$ has been estimated from the measurements of Campbell and DeBenedetti on $\mathrm{NH}_{4}(\mathrm{AlFe})\left(\mathrm{SO}_{4}\right)_{2}$ - $12 \mathrm{H}_{2} \mathrm{O},{ }^{17,21}$ which showed a magnetic field of $H_{\max }=590 \mathrm{kG}$ for the $\frac{5}{2}$ state, in good agreement with the results of Bruckner et al. ${ }^{19,20}$ on highly magnetized diluted and undiluted ferric alum, which indicated $H_{\max }=595 \pm 10 \mathrm{kG}$. In the actual temperature range the average magnetic field at the place of the iron nuclei can be neglected since the external field is partly compensated by the induced average hyperfine field. The quadrupole splitting was assumed to be zero. Examples of the fitting are shown in Figs. 2 and 3.

Figure 2 shows spectra obtained at $120 \mathrm{~K}$ in a 5-kG external field parallel and perpendicular to the $\gamma$-ray direction. The successful fitting with intensity ratios 3:0:1 and 3:4:1, and a common spin-correlation time $\tau=1.1 \mathrm{nsec}$ seems to justify the application of the simple model.

In Fig. 3 are shown two typical spectra obtained in a 5-kG perpendicular field at 175 and $89 \mathrm{~K}$. The full lines indicate the theoretical spectra.

In Fig. 4 the estimated values of $\tau$ are shown as a function of the temperature. The temperature dependence of the correlation time is evident. In the actual temperature range and with a field of $5 \mathrm{kG}$ the populations of the ionic states are almost constant. Hence $\tau_{\text {ss }}$ should be almost constant. Therefore, the temperature dependence must be due to spin-lattice interactions. Even if $\tau_{s 1}$ is assumed to be long for $S$-state ions like $\mathrm{Fe}^{+3}$, this result is not surprising in the light of measurements on $\mathrm{NH}_{4}(\mathrm{Fe}, \mathrm{Al})\left(\mathrm{SO}_{4}\right)_{2} \cdot 12 \mathrm{H}_{2} \mathrm{O}, 16,17,21$ indicating a temperature-dependent spin-correlation time.

\section{DISCUSSION}

It is interesting to compare our results with the existing theories for spin-lattice processes in $S$-state compounds. We find that the slope of $\tau$ versus temperature is more pronounced below the Debye temperature $\Theta_{D}$ than above. $\Theta_{D}=134 \mathrm{~K}$ was derived from the temperature dependence of the integral intensity of the Mössbauer absorption line. These observations agree well with the theories. ${ }^{33,34}$ A separation of $\tau_{s s}$ and $\tau_{s 1}$ could in principle have been made on the basis of one of the models by Suzdalev et al. ${ }^{33}$ This has been tried but the results were considered doubtful as none of the models agreed in detail with our measurements. The deviation can be due to the simplicity of the models. The theories only deal with transitions induced by modulation of a temperature-independent crystal field in the absence of external fields. However, the crystal-field parameters vary as a function of temperature $\mathrm{e}^{27,28}$ and furthermore our results are obtained in a 5kG field.

Gleason and Walker ${ }^{22}$ have measured the linewidth and line area of Mössbauer spectra of ferric alum in zero applied field. The linewidth was found to depend in a complicated way on the temperature. Above $140 \mathrm{~K}$ the linewidth was found to increase with temperature. This was considered to be due to an increasing spin-correlation time contrary to our results in the same temperature range. A more pronounced increase of the linewidth as function of the temperature in a 700-G external field has later been found. ${ }^{35}$ However, these results cannot directly be compared with ours as we have applied a 5-kG external field. It is uncertain whether the linewidth in zero and low fields can be related to the spin-correlation time since transverse relaxation may complicate the situation considerably. The crystal field, as well as an external magnetic field, gives rise to a more or less strong polarization of the ferric ions. Therefore, a change in the crystal-field parameters may influence the Mössbauer spectra in a way similar to a change in the magnitude of the external field. ESR measurements ${ }^{27,28}$ have 
shown a strong temperature dependence of the crystal-field parameters although the total splitting is limited to about $0.1-0.2 \mathrm{~K}$. This could be the explanation of the above-mentioned data obtained in 0 and $700 \mathrm{G}$.

\section{CONCLUSION}

We have found that Mössbauer spectroscopy is a useful tool for measurements of spin-correlation times in undiluted ferric compounds when computer fitting is used. The simple model used gives results in good agreement with experiments.

The measured spin-correlation times give information about the spin fluctuations which cannot be found from bulk measurements. The low-field spectra are affected by fluctuations both parallel and perpendicular to the $z$ direction. The detailed line shape has not yet been calculated for this case.

It has been shown clearly that spin-lattice interactions play an important role in ferric alum and the temperature dependence of the spin-lattice interaction is in qualitative agreement with theory.

\section{ACKNOWLEDGMENT}

We are grateful to Professor G. Trumpy for encouragement and continuous interest during this work.
${ }^{1}$ A. M. Afanas'ev and Yu. Kagan, Zh. Eksperim. i Teor. Fiz. $\underline{45}, 1660$ (1963) [Sov. Phys. JETP $\underline{18}, 1139$ (1964)].

${ }^{2}$ E. Bradford and W. Marshall, Proc. Phys. Soc. (London) 87, 731 (1966).

${ }^{3}$ H. Gabriel, Phys. Status Solidi 23, 195 (1967).

${ }^{4}$ H. H. Wickman, M. P. Klein, and D. A. Shirley, Phys. Rev. 152, 345 (1966).

${ }^{5}$ H. Wegener, Z. Physik 186, 498 (1965).

${ }^{6} \mathrm{H}$. H. Wickman and G. K. Wertheim, in Chemical Applications of Mössbauer Spectroscopy, edited by V. I. Goldanskii and R. H. Herber (Academic, New York, 1968) (in this review article other references can be found).

${ }^{7} \mathrm{M}$. Blume and J. A. Tjon, Phys. Rev. $\underline{165}, 446$ (1968).

${ }^{8} \mathrm{~F}$. van der Woude, thesis (University of Groningen, Netherlands, 1966) (unpublished).

${ }^{9}$ B. C. van Zorge, W. J. Caspers, and A. J. Dekker, Phys. Status Solidi 18, 761 (1966).

${ }^{10} \mathrm{H}$. Gabriel, J. Bosse, and K. Rander, Phys. Status Solidi 27, 301 (1968).

${ }^{11}$ M. A. Krivoglaz and V. F. Los, Phys. Status Solidi 42, 71 (1970).

${ }^{12}$ H. Schwegler, Phys. Status Solidi $\underline{41}, 353(1970)$.

${ }^{13}$ J. W. G. Wignall, J. Chem. Phys. 44 , 2462 (1966).

${ }^{14}$ R. M. Housley and H. de Waard, Phys. Letters 21 , 90 (1966).

${ }^{15}$ R. M. Housley, J. Appl. Phys. 38, 1287 (1967).

${ }^{16} \mathrm{H}$. de Waard and R. M. Housley, in Hyperfine Interactions, edited by A. J. Freeman (Academic, New York, 1967), p. 691.

${ }^{17}$ L. E. Campbell and S. DeBenedetti, Phys. Rev. 167, 556 (1968).

${ }^{18}$ F. E. Obenshain, L. D. Roberts, C. F. Coleman, D. W. Forester, and J. O. Thomson, Phys. Rev. Let- ters 14,365 (1965).

${ }^{19}$ W. Bruckner, G. Ritter, and H. Wegener, Z. Physik 200,421 (1967).

${ }^{20}$ W. Bruckner, G. Ritter, and H. Wegener, Z. Physik 236, $52(1970)$.

${ }^{21}$ L. Campbell and S. DeBenedetti, Phys. Letters $\underline{20}$, 102 (1966).

${ }^{22}$ T. G. Gleason and J. C. Walker, Phys. Rev. $\underline{188}$, 893 (1969).

${ }^{23}$ A. M. Afanas'ev and Yu. M. Kagan, Zh. Eksperim. i Teor. Fiz. Pis'ma v Redaktsiyu $\underline{8}, 620$ (1968) [Sov. Phys. JETP Letters $\underline{8}, 382$ (1968)].

${ }^{24}$ V. D. Gorobchenko, I. I. Lukashevich, V. V. Sklyarevskii, K. F. Tsitskishvili, and N. I. Filippov, Zh. Eksperim. i Teor. Fiz. Pis'ma v Redaktsiyu $\underline{8}$, 625 (1968)) [Sov. Phys. JETP Letters 8 , 386 (1968)].

${ }^{25} \mathrm{~J}$. Vogler, F. W. de Vrijer, and C. J. Gorter, Physica 13 , 621 (1947).

${ }^{26}$ P. H. E. Meijer, Physica 17, 899 (1951).

${ }^{27}$ S. Maekawa, J. Phys. Soc. Japan 16, 2337 (1961).

${ }^{28}$ S. Maekawa, J. Phys. Soc. Japan 17, 1208 (1962).

${ }^{29} \mathrm{~S}$. Mфrup and N. Thrane, in Proceedings of the International Interactions Detected by Nuclear Radiation, Israel, 1970 (unpublished).

${ }^{30} \mathrm{H}$. de Waard (private communication).

${ }^{31} \mathrm{~N}$. Thrane and G. Trumpy, Phys. Rev. B 1,153 (1970).

${ }^{32}$ M. Blume, Phys. Rev. Letters 18, 305 (1967).

${ }^{33}$ I. P. Suzdalev, A. M. Afanas'ev, A. S. Plachinda, V. I. Goldanskii, and E. F. Makarov, Zh. Eksperim. i Teor. Fiz. $\underline{55}, 1752$ (1969) [Sov. Phys. JETP 28, 923 (1969)].

${ }^{34}$ V. V. Svetozarov, Fiz. Tverd. Tela 12, 1054 (1970) [Sov. Phys. Solid State 12, 826 (1970)].

${ }^{35}$ Boye Olsen (private communication). 